UNITED STATES DEPARTMENT OF THE INTERIOR

GEOLOGICAL SURVEY

Developing Solenoids for use with Magnetic

Susceptibility We11-Logging Systems

By

Joseph J. Barth, James H. Scott and Robert L. Seeley

Open-File Report

$86-115$

This report is preliminary and has not been edited or reviewed for conformity with U.S. Geological Survey Standards and nomenclature. 


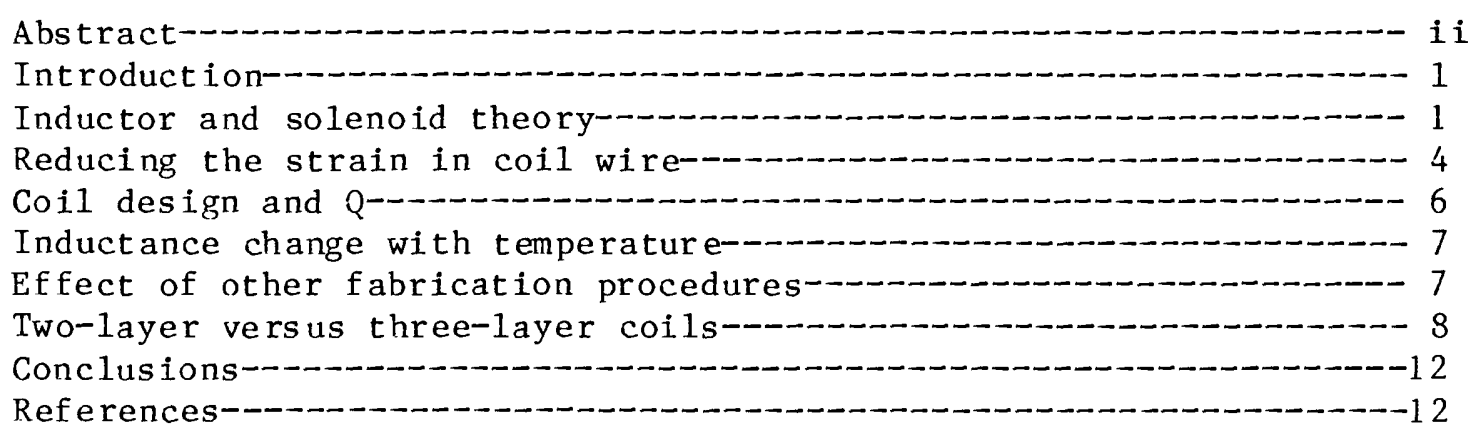

\section{ILLUSTRATIONS}

Figure 1. Solenoid and flux lines- 2

2. Initial permeability vs. temperature for ferrite core material MN-67- 4

3. Solenoid inductance vs. temperature for (A) uncoated ferrite, and (B) RTV-coated ferrite----- 5

4. Current of a series-resonant circuit as a function of frequency- 6

5. Solenoids with double-layer coils: (A) MN-30, and (B) $\mathbb{M N}-67-\ldots$

6. Triple-layer solenoid coil pattern-

7. Solenoid inductance vs. temperature for (A) doublelayer coil, MN-30, and (B) triple-layer coil, $\mathrm{MN}-67-\ldots$ 


\title{
DEVELOPING SOLENOIDS FOR USE WITH MAGNETIC SUSCEPTIBILITY WELL LOGGING SYSTEMS
}

\author{
By \\ Joseph J. Barth, James H. Scott, and Robert L. Seeley \\ U.S. Geological Survey, Denver, Colorado 80225
}

\begin{abstract}
The solenoid, employed as the inductive sensor of a Maxwell bridge circuit, is one of the key components of a magnetic susceptibility welllogging system for mineral exploration. Solenoids exhibit changes of inductance with changes of temperature. Fabrication techniques have been developed that reduce these effects over a limited temperature range. Solenoid theory and its application to construction methods that improve the sensitivity and temperature characteristics of solenoids are discussed in this report. The coil design, the effects of strain on the wire generated by the winding process, coil dimension changes and fabrication methods that deal with these characteristics are presented. Problems associated with coil heater design, including the effects of placing metal objects close to the coil are discussed. The advantages of using high inductance, high $Q$ solenoids in magnetic susceptibility systems are presented in theory and application. Multilayer coils inherently contain irregular characteristics, (Terman, 1943). Techniques that overcome these characteristics or use them to advantage are explained. Construction data from 3-layer solenoids having approximatly twice the inductance of previously used USGS solenoids are included for the reader's information.
\end{abstract}




\section{INTRODUCTION}

The magnetic susceptibility of rocks containing ore-grade concentrations of ferromagnetic minerals is high, therefore, low sensitivity magnetic susceptibility tools may be used to detect these anomalies with good results. As the ferromagnetic mineral content decreases however, the measurement becomes increasingly difficult because of noise and drift due to temperature variation and mechanical stress of components in the measuring circuit. Various experiments have indicated that these effects may be reduced appreciably by using components having a high degree of temperature stability in the measuring circuit of these tools.

The sensing coil, constructed in the form of a solenoid, and used as part of the Maxwell bridge measuring circuit, is temperature sensitive, but unlike other components it is not commercially available and must be customfabricated for each magnetic susceptibility tool. The solenoid must have an inductance that is sufficiently high $(50-100 \mathrm{mH})$ to detect changes in susceptibility as small as one micro cgs unit, and must exhibit little or no inductance change with temperature over the operating temperature range. Enclosing the solenoid in a temperature controlled chamber limits the operating temperature range, but even a small change in temperature will affect measurements if temperature dependence of the solenoid's inductance is not reduced.

A typical magnetic susceptibility system's usefulness, as an anomaly detecting tool depends on the sensitivity and temperature stability of the sensing solenoid and therefore it is important that all procedures that might affect them be carefully examined before and during fabrication. This report discusses some theories and fabrication techniques used to improve the performance of solenoids used in magnetic susceptibility systems.

\section{INDUCTOR AND SOLENOID THEORY}

If inductor theory is overlooked in the construction of magnetic susceptibility solenoids, problems may occur that limit the success of the solenoid application. The following theory is considered important to solenoid design, and is therefore included for the reader's information.

An inductor is a device for storing energy in a magnetic field. Loops, coils, and solenoids are inductors. The lines of magnetic flux produced by a current in a solenoidal coil form closed loops as seen in figure 1 (Kraus, 1953, p. 164). 


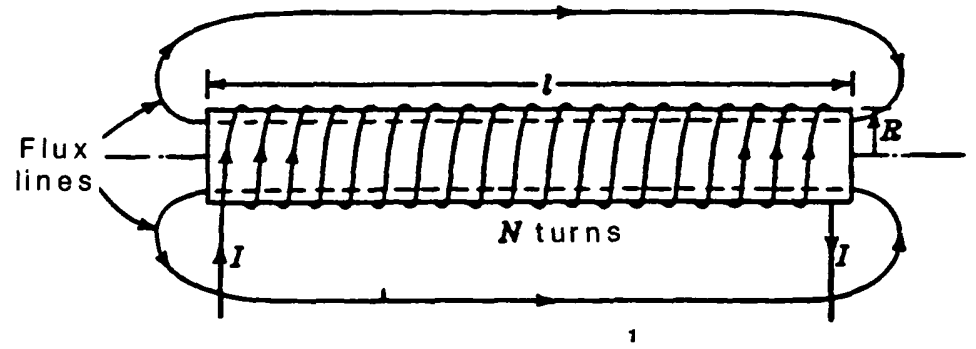

Figure 1.--Solenoid and flux lines.

It is said that each flux line that passes through the entire solenoid as in the figure links the current $N$ times. "If all of the flux lines link all of the turns, then the total magnetic flux linkage $\Lambda$ of the coil is equal to the total magnetic flux $\psi$ through the coil times the number of turns..." (Kraus, 1953, p. 164).

$$
\Lambda=\mathrm{N} \psi
$$

where $\Lambda$ is flux linkage and $\psi$ is magnetic flux.

Since the number of turns, $N$, is dimensionless, flux linkage has the same demensions as flux. By definition, "the inductance, $L$, of an inductor is the ratio of its total magnetic flux linkage to the current, I, through the inductor..." (Kraus, 1953, p. 164).

$$
\mathrm{L}=\frac{\mathrm{N}_{\psi}^{\prime}}{\mathrm{I}}=\frac{\Lambda}{\mathrm{I}}
$$

It can be seen from the equation that if the inductance of a solenoid is doubled, the flux density will also double if current is held constant.

The preceding equation is valid for coils having air cores; for coils with ferromagnetic cores the inductance is more correctly defined as, "...the ratio of infinitesimal change in flux linkage to the infinitesimal change in current producing it," (Kraus, 1953, p. 165).

$$
\mathrm{L}=\frac{\mathrm{d} \Lambda}{\mathrm{dI}}
$$


The inductance of solenoids may be calculated from their geometry. However, an approximation must be made because the flux density at the end of a long solenoid is less than at the center due to flux leakage inside the ends of the solenoid. If the solenoid is very long, one may assume the flux density to be nearly constant over the entire interior of the solenoid and nearly equal to its value at the center (Kraus, 1953, p. 165).

The inductance of a long solenoid may be calculated using:

$$
L=\frac{\Lambda}{I}=\frac{\mu N^{2} A}{1}
$$

where $\mathrm{L}$ is the inductance of solenoid (henrys), $\Lambda$ is flux linkage (weber turns), I is current through solenoid (amperes), $\mu$ is the permeability of medium (henrys/meter), $\mathrm{N}$ is the number of turns of the solenoid

(dimensionless), A is cross-sectional area of solenoid (meters ${ }^{2}$ ), and 1 is the length of solenoid (meters) (Kraus, 1953, p. 165).

It can be seen from the equation above that the inductance, $L$, increases in proportion to an increase in either the permeability, $\mu$, or the crosssectional area, A, of the solenoid. The number of turns in the coil winding, $\mathrm{N}$, is significant because the inductance increases as the square of $\mathrm{N}$. Therefore, the most effective way to increase the solenoid sensitivity assuming cross sectional area and current remain the same is to add more turns to the coil. As more turns are added, however, the temperature dependence usually increases as well. Given a fixed cross sectional area of winding, increasing the number of turns in the coil also increases the resistance of the coil so that there is no increase in the coil's Q. Actually, experiments have shown the $Q$ of the coil will decrease slightly. The $Q$ is the ratio of the inductive reactance to the resistance. The effects of changing the $Q$ of the solenoid will be explained later in the paper.

An air-core solenoid does not yield enough inductance to be useful in a high-sensitivity magnetic susceptibility tool. Therefore, a ferromagnetic core of a high-permeability material with stable temperature characteristics is more commonly used.

The permeability, $\mu$, of a substance is defined as

$$
\mu=\frac{B}{H}=\mu_{0}{ }^{\prime} r
$$

where $B$ is the flux density (webers/meter ${ }^{2}$ ), $H$ is the magnitude of the magnetic field (amp/meter), $\mu_{0}$ is the permeability of a vacuum ( $4 \times 10^{7}$ amp/meter), and the ${ }_{1} \mathrm{r}$ relative permeability of the substance (dimensionless), (Kraus, 1953, p. 232).

When choosing a core material one should examine the initial permeability versus temperature curve in the manufacturer's specifications. 


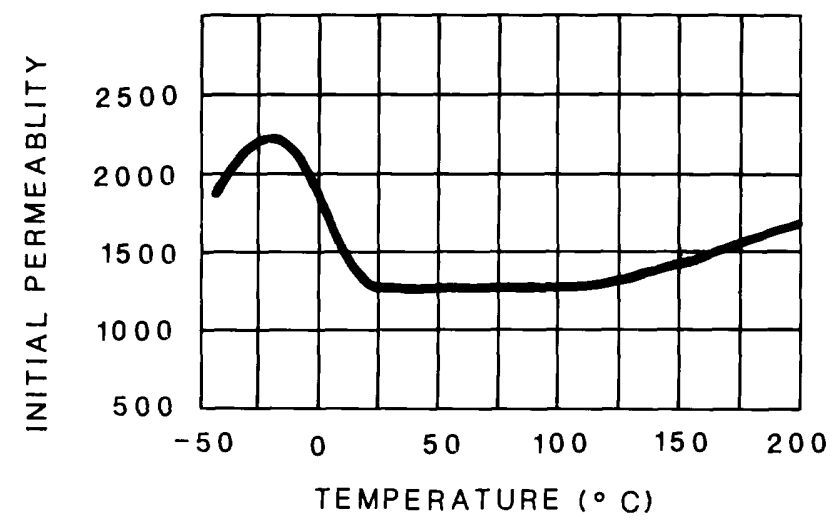

Figure 2.--Initial permeability vs. temperature for ferrite core material $\mathrm{MN}-67$.

Figure 2 shows a plot of the initial permeability versus temperature of the ferrite MN-67 manufactured by Ceramics Magnetics Inc. ${ }^{1}$ As implied by figure 2, solenoids wound on ferrite cores exhibit the least inductance change with temperature when the temperature of the solenoid is controlled within the range of the flat part of the curve, in this case from $25^{\circ} \mathrm{C}$ to $100^{\circ} \mathrm{C}$.

\section{REDUCING THE STRAIN IN COIL WIRE}

According to Terman (1943, p. 85), variation of inductance with temperature of solenoids is caused mainly by mechanical strain in the coil wire introduced during the winding process. He reasoned that if the wire was allowed to flex more easily on the form on which the coil was wound, the strain would be less and therefore the inductance change with temperature would be less. To try to duplicate this condition, we applied heat shrink tubing, paints, silicone rubber and adhesive tapes in separate experiments, to the surface of the ferrite to create a flexible surface on which to wind the coil. In a typical experiment, two layers of $0.792 \mathrm{~mm}$ copper wire were bank wound over a Ceramic Magnetics $M N 30$ ferrite rod, $1.5 \mathrm{~cm}$ in diameter and $30 \mathrm{~cm}$ long. The inductance was measured at $2^{\circ} \mathrm{C}$ increments with a bridge at a frequency of $1000 \mathrm{~Hz}$ over a temperature range of $50^{\circ} \mathrm{C}$ to $100^{\circ} \mathrm{C}$. Next, the coil wires were removed and a coating of RTV silicone rubber about $1 \mathrm{~mm}$ thick was applied to the surface of the ferrite rod. After the RTV had cured, another identical coil was bank wound over the RTV coating and the inductance of the second coil was measured over the same temperature range as before. Figure 3 shows the temperature characteristics of two solenoids, each measured on a bridge with an input frequency of $1000 \mathrm{~Hz}$. Comparing trace A (uncoated) with the trace B (RTV-coated) it can be seen that the RTV coating degraded the temperature characteristics of the solenoid. All other coatings applied to

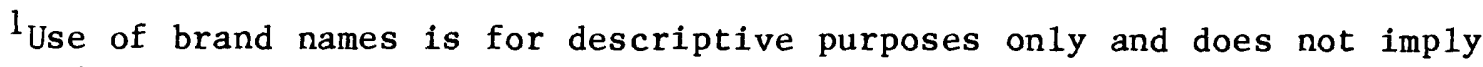
endorsement by the USGS. 
the ferrite surface prior to the coil winding produced essentially the same irregular results as trace $B$ of figure 3. The experiments indicate that if any material is placed between the coil and the ferrite, the inductance of the solenoid becomes more dependent on temperature.

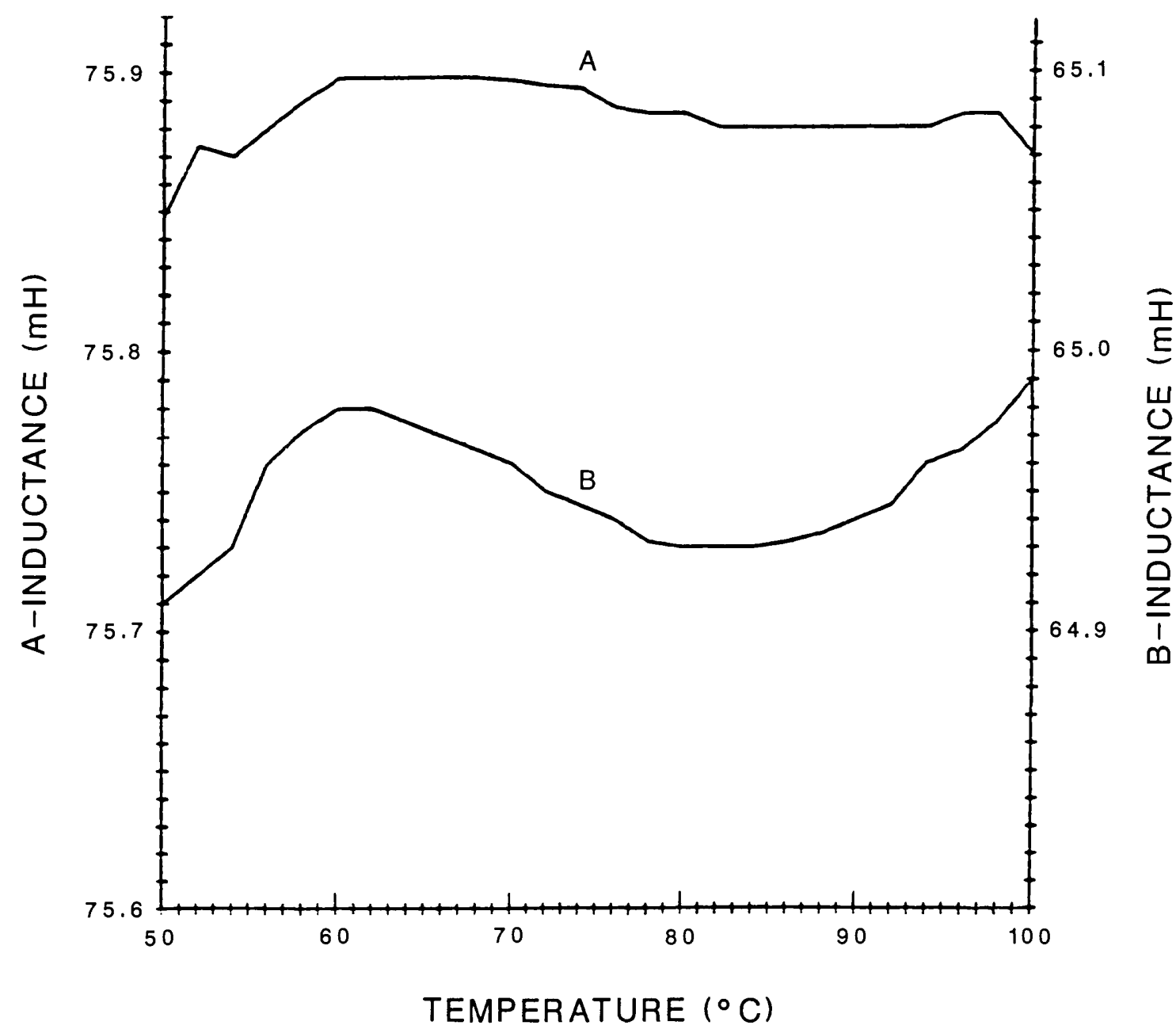

Figure 3.-- Solenoid inductance vs. temperature for

(A) uncoated ferrite, and (B) RTV-coated ferrite.

Another approach to reducing the strain in the coil wire involves prebending the wire to the shape of the ferrite form (suggested by Terman, 1943, p. 86) by winding the wire on a wood dowel about $3 / 4$ the diameter of the ferrite rod. The objective is to release the tension of the wire while on the dowel, letting it spring back to the shape of the ferrite rod and then to slip it sideways off of the dowel and onto the ferrite rod. The results of this painstaking process proved to be no better than the coated ferrite core experiment shown in figure 3. 
Similarly, relieving strain by annealing the coil in an oven did not help stabilize the inductance change with temperature when this technique was used in conjunction with the technique mentioned above. Furthermore, to achieve any significant strain relief the temperature had to be raised to the point that the process destroyed the insulation normally coating the copper wire, and thus, shorted the turns of the coil.

\section{COIL DESIGN AND Q}

The $Q$ or "quality" of the coil is defined as the radio of coil reactance to coil resistance:

$$
Q=\frac{X_{L}}{R}
$$

The reactance of a coil is given by the formula

$$
X_{L}=2 \pi f L
$$

where $\mathrm{f}=$ frequency and $\mathrm{L}=$ inductance.

In the construction of a solenoid, it is usually desireable to attain a low coil resistance, hence a high $Q$, because this reduces the current losses. Current losses reduce the dynamic response of the bridge circuit when at balance. Figure 4 shows that increasing the circuit resistance reduces the circuit response at the resonant frequency (Terman, 1943, p. 135).

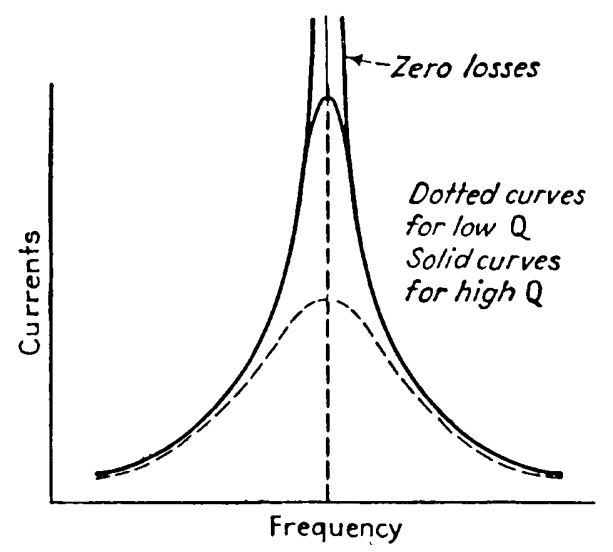

Figure 4.--Current of a series-resonant circuit as a function of frequency (from Terman, 1943). 
The data acquired from coil experiments lead us to conclude that the strain in a coil of wire produced by the winding procedure is not the major cause of a solenoid's inductance change with temperature although it may produce a second-order effect. Apparently the most significant cause of a solenoid's inductance change with temperature is another factor suggested by Terman (1943, p. 85), "the change of dimensions of the coil wires and the ferrite rod with changes in the temperature".

We have found that this phenomenon can be turned to an advantage, if proper construction techniques are used. When a copper wire is wound over a ferrite rod, a mismatch of the rates of expansion of the materials is present. The copper wire has a thermal coefficient of expansion of $16 \times 10^{-60}$ $\mathrm{C}$ compared to most ferrites which are $1-11 \times 10^{-60} \mathrm{C}$. The copper expands at a greater rate than most ferrites, but with experimentation it was found that if the copper wire is wound with just the right amount of tension, at some temperature the change of inductance with temperature will be at a minimum and remain minimum over a range of several degrees centigrade. It is possible to create this condition by experimenting with the tension of the wire during the winding process until the minimum inductance change with temperature occurs within the flat interval of the initial permeability versus temperature curve (see figure 2 ) of the ferrite material selected.

Simply winding a coil on a ferrite rod with tension applied to the copper wire does not necessarily produce the results mentioned above. Several other factors affect the process of obtaining a minimum inductance change with temperature in a solenoid. The type and size of the ferrite material, the size of the copper wire, the number of windings and layers of the coil, as well as the tension applied to the wire and possibly other factors, all affect the temperature characteristics of the solenoid (Terman, 1943, p. 76). Developing a coil-winding technique that uses these parameters to advantage is a matter of trial and error. If a coil is wound with little or no tension, the results obtained will be similar to the irregular response of the coated ferrite (trace B) shown in figure 3. However, if a moderate amount of tension is applied, the response is smoother and a minimum change in inductance occurs at some temperature. Once the minimum inductance change with temperature point has been established for a particular coil configuration, experimentation has indicated that applying less tension to the wire during the winding process will shift the minimum inductance change point to a slight1y lower temperature and result in a wider span in the usable temperature range. Conversely, if more tension is applied, the minimum inductance change point will be shifted to a slightly higher temperature, resulting in a narrower span of the temperature range. The exact tension to be applied in the winding process is difficult to determine and several trial and error iterations are usually required. The tension used on the coils that we have wound ranged from approximately 1 to 3 pounds.

\section{EFFECT OF OTHER FABRICATION PROCEDURES}

After the coil winding process is completed and the desirable inductance versus temperature characteristics have been established, it is important that they are not degraded by the additional steps needed to complete the fabrication of the system. In our early experiments, solenoids were 
constructed that initially exhibited acceptable inductance versus temperature characteristics that were subsequently degraded by applying epoxy, or by attaching a metal shield directly to the surface of the coil wires. The coil surface may be sealed with a thin application of RTV silicone rubber. Materials that are hard when cured should not be used for sealing the coil because they introduce stress by differential thermal expansion. The solenoid should be supported only on each end of the ferrite rod with flexible mounts such as spongy plastic. Rigid materials that might introduce stress to the coil or to the ferrite rod should not be used. The solenoid is an extremely stress-sensitive device and one must keep this fact in mind from start to finish during the fabrication procedure. It is important that the desirable minimum inductance change with temperature characteristic is retained and not degraded by the mounting technique. Metal objects such as a Faraday shield, should not be located close to a coil because eddy currents will be induced in the object that will tend to oppose the change in the field of the coil (Kraus, p. 315).

Thermoregulation of the solenoid is usually achieved by controlling current through resistors and heating The solenoid to a temperature greater than temperature that will be encountered in a borehole (Scott and others, 1981 , p. $(C 1-\operatorname{CC} 21)$.

Attaching heater resistors directly to the coil surface introduces a magnetic field to the coil when current flows through the heater string. This undesireable effect is even more pronounced if the string of resistors is constructed in the form of a loop (Stout, 1960, p. 319). The location of heater elements or an electrostatic shield should ideally be no closer than a distance "...at least equal to the radius of the coil..." (Terman 1943, p. 128). This may not be possible given the size restrictions of borehole tools, but in this case the heater and shield should be located as far away from the coil surface as is practical.

\section{TWO-LAYER VERSUS THREE-LAYER COILS}

Until recently, only one-and two-layer coils have been made by the USGS for solenoids used in magnetic susceptibility tools. In early tests, treelayer solenoid prototypes were wound on available ferrite cores in hopes of increasing the inductance beyond that of the two-layer solenoid. However, the temperature dependence results were disappointing and experiments were discontinued at that time.

More recent experiments with different coil designs have indicated that each type and size of ferrite rod produces a unique inductance versus temperature curve with a particular coil configuration. Figure 5 compares two double-layer solenoids using Ceramics Magnetics, MN-67 (A) and MN-30 (B) ferrites. 


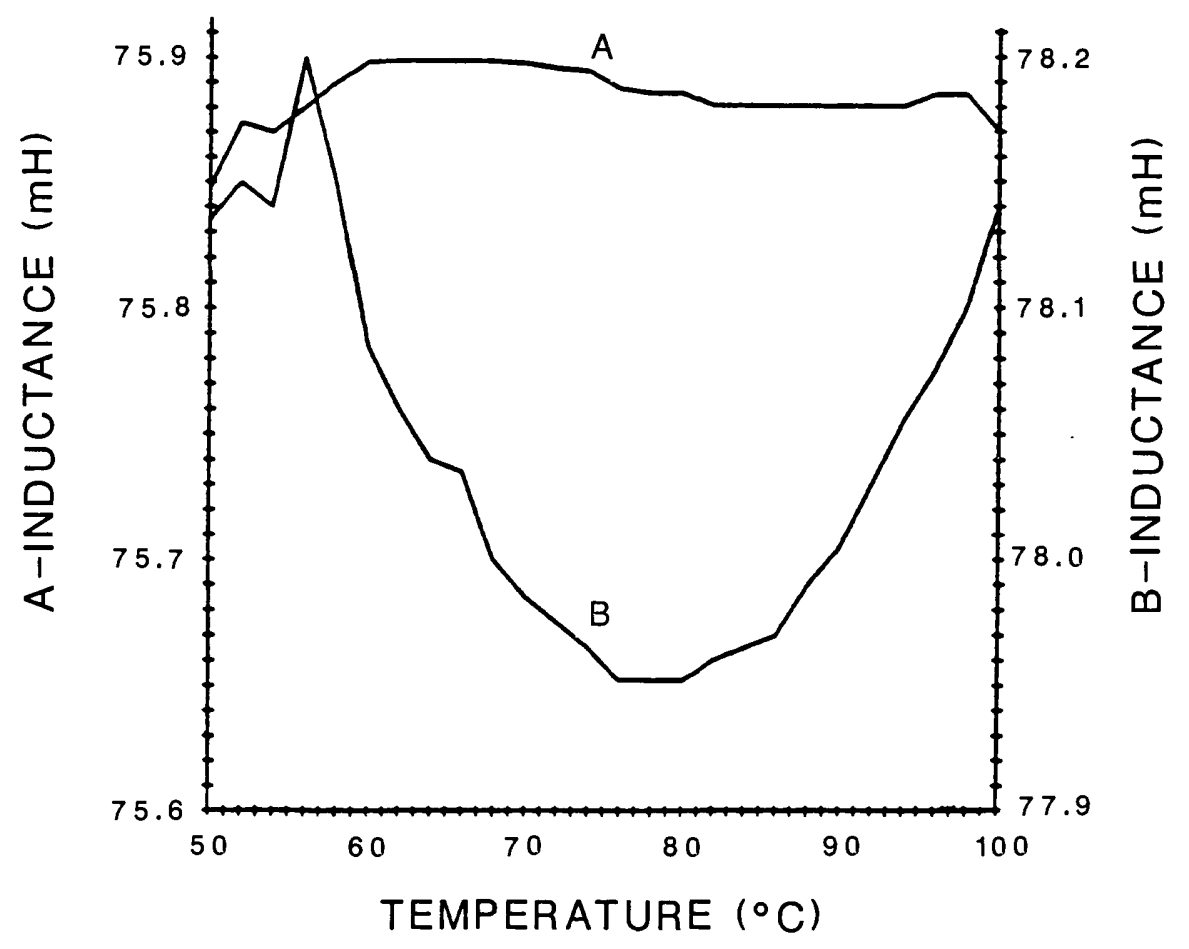

Figure 5.--Solenoids with double-layer coils: A, MN-30, and B, MN-67.

The MN-30 ferrite used in conjunction with fabrication techniques mentioned above has produced excellent 2 layer solenoids and has been used with good results in USGS magnetic susceptibility tools. The following data were found to be typical for an MN-30 double layer solenoid whose temperature response is plotted in figure 5, trace A.

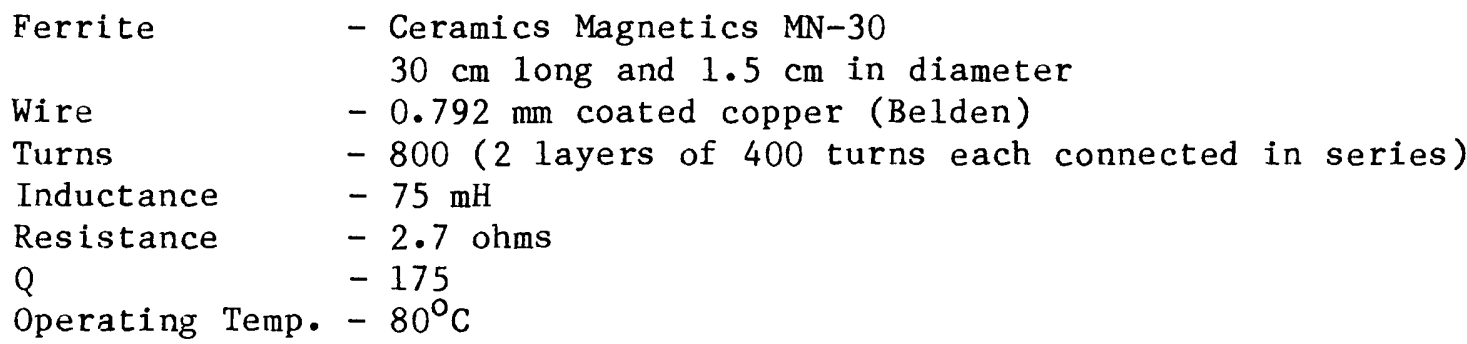

Recently, the theory and the technique of coil construction used by others was re-examined to determine the feasibility of using triple layered coils in solenoids. Terman (1943, p. 84) tells us that "multilayer coils tend to have higher distributed capacity..." To reduce this effect, "...the winding should be a "bank winding" that is, each consecutive winding layer should be wound in the grooves of the previously wound coil. This reduces the space between windings and that reduces the distributed capacity (Terman 1984, p. 84). Additionally, the wires should not be crossed when winding a coil because crossed windings will also add distributed capacity.

The presence of distributed capacity introduces losses that have the same effect as adding resistance in series with the coil (Terman, 1943, p. 84) but at an operating frequency of $1000 \mathrm{~Hz}$ it is most likely not significant. The 
inductance of a multilayer coil increases as the square of the number of turns, but as inductance increases, undesirable temperature effects increase as well. Terman describes the noncyclical variation of coil inductance with temperature change and tells us, "... if the coil undergoes temperature cycles the inductance will not follow the temperature exactly, but, rather, will vary irregulary...." "This is apparently the result of mechanical changes such as result from slippage of the wire over the form, and permanent changes in physical dimensions..." (Terman, 1943, p. 86).

Thus, increasing the number of turns of wire will also increase the "irregular" properties making it more difficult to minimize temperature dependence in multilayer solenoids. However, a high Q, triple-layer solenoid having a limited useful temperature span has been developed by the USGS using the construction techniques described previously for two-layer coils. The solenoid is made by bank winding three consecutive coils, one over the other, and then connecting one end of each layer to the opposite end of the adjacent layer (see figure 6).

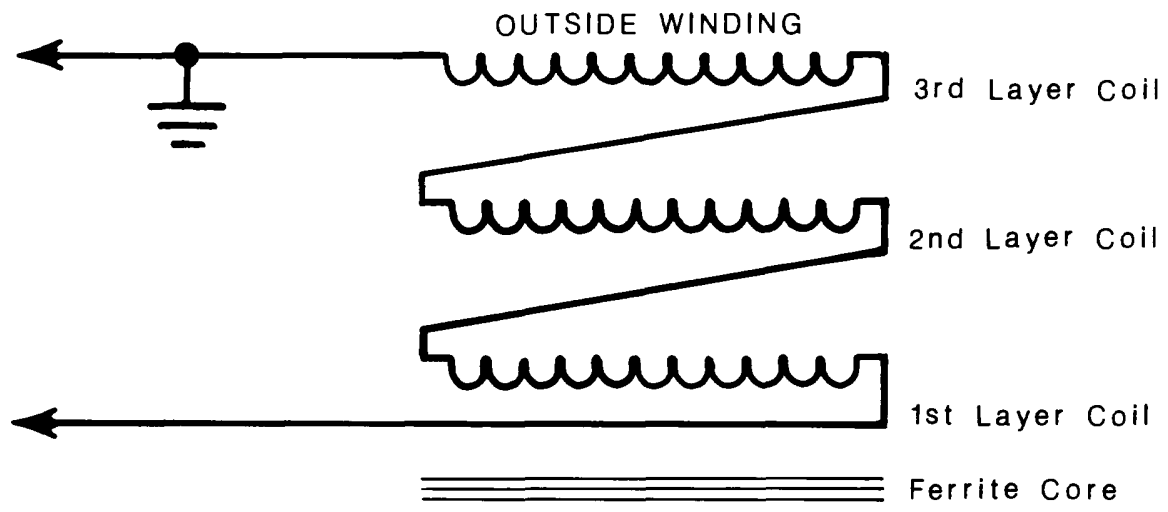

Figure 6.--Triple-1ayer solenoid coil pattern.

All multilayer coils are connected in a bank-wound configuration as shown in figure 6 because attaching coil wires together from the same end of the solenoid would critically lower the solenoid's inductance. solenoid.

Figure 7 compares a USGS double-layer solenoid with a triple-layer 


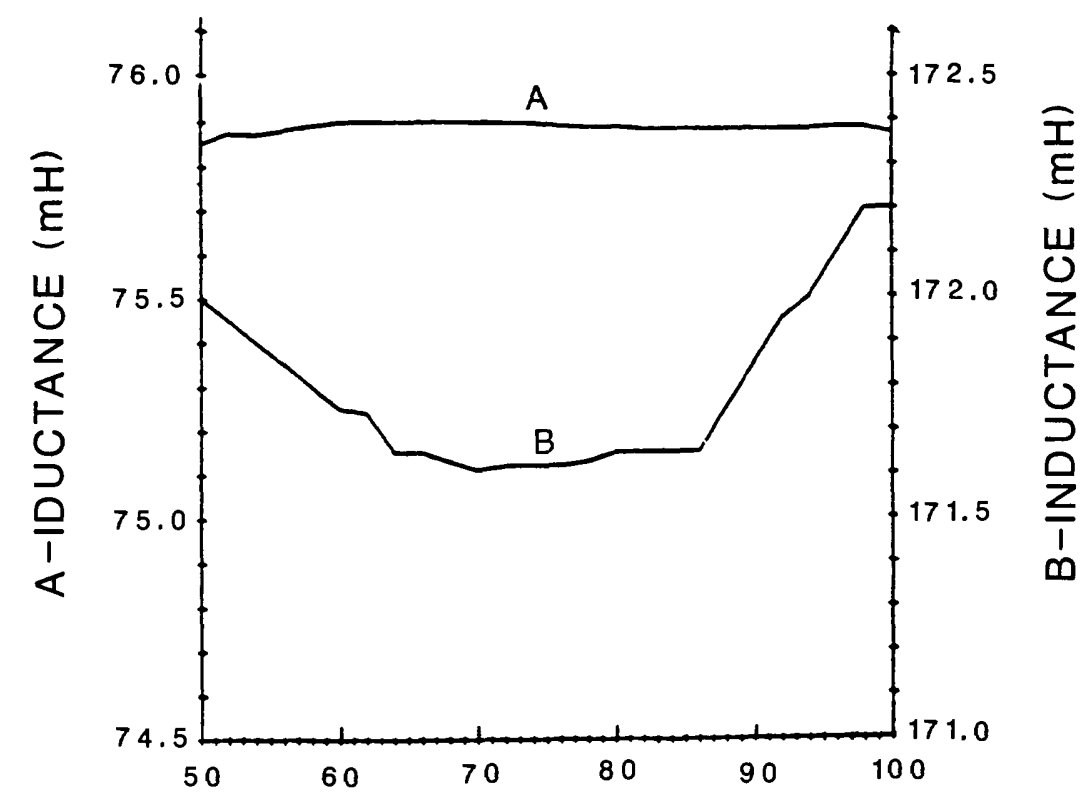

Figure 7.-- Solenoid inductance vs. temperature for (A) double-1ayer coil, $\mathrm{MN}-30$, and (B) triple-1ayer coil $\mathrm{MN}-67$.

Notice that the triple-layer solenoid, constructed with MN-67 ferrite core, has about twice the inductance of the best double-layer solenoid constructed with MN-30 ferrite. Also, the $Q$ of the double-layer solenoid in this case, is 175 while the triple-layer solenoid has a $Q$ of 240 . The sensitivity of the triple layer solenoid did not increase proportionately with the inductance, which was probably due to the lower permeability of MN-67 (about half that of $\mathrm{MN}-30$ ). Also, the triple-layer solenoid is more temperature dependent and therefore, would not be as useful over the same range of temperatures as the $\mathrm{MN}-30$ double-layer solenoid. It should be noted that the three-layer solenoid is still considered experimental, although it was used successfully for making a susceptibility $\log$ of Hole 418A in old oceanic crust south of Bermuda (Leg 102 Scientific Party, 1985). It was not intended to replace the standard two-layer solenoid but rather, with additional experimentation using other core materials, it might be perfected as a sensitive device for special applications where low-level susceptibilities are to be measured.

The three-layer solenoid has the following specifications and characteristics:

Ferrite core material

Ferrite core size

Wire

Turns

Inductance

Resistance

Q

Operating temperature
Ceramics Magnetics $\mathrm{MN}-67$

$1.5 \mathrm{~cm}$ in diameter, $30 \mathrm{~cm}$ in length

$0.792 \mathrm{~mm}$ coated copper (Belden)

1200,3 layers in series

$174 \mathrm{mH}$

4.5 ohms

240

$80^{\circ} \mathrm{C}$ 


\section{CONCLUSIONS}

Solenoids, used as sensors in magnetic susceptibility systems have been developed by the USGS that exhibit little temperature dependence over a limited temperature range. The application of solenoid theory, combined with the selection of compatible core materials and the experimentation of coil winding techniques, have resulted in the reduction of distributed capacity and other undesireable effects. The effects of thermal expansion of coil wires, as explained by Terman (1943), combined with improved coil winding techniques, have been used to advantage for developing two-layer solenoids with a low temperature dependance. Advances in the above techniques have made possible the construction of experimental high $Q$, three-layer solenoids that may find use in future applications where sensitive, limited temperature range solenoids are required.

\section{REFERENCES}

Kraus, John D., 1953, Electromagnetics: New York, McGraw-Hill Book Co., p. $143-166,206-240$.

Leg 102 Scientific Party, 1985, 01d hole yields ew information: Geotimes, v . 30 , no. 12 , p. 13-15.

Scott, J.H., Seeley, R. L., and Barth, J. J., 1981, A magnetic susceptibility we11-logging system for mineral exploration: Transactions, SPWLA 22nd Annual Logging Symposium, June 23-26, 1981, Mexico City, Mexico, p. CClCC21.

Stout, M. B., 1963, Basic electrical measurements: second edition, Englewood Cliffs, N.J., Prentice-Hall, Inc., p. 319-322.

Terman, F. E., 1943, Radio engineers handbook: New York, Mccraw Hi11, p. 85$92,128-134$. 